\title{
La intervención con Menores Expuestos a Violencia de Género en los Puntos de Encuentro Familiar. Disfuncionalidades y retos en la Comunidad Valenciana
}

\section{Intervention with Children Exposed to Gender Violence in the Family Meeting Points. Dysfunctions and challenges in Valencian Community}

Fecha de recepción: 08/04/2016

Fecha de aceptación: 28/04/2016

Antonio Marín Manrique

Licenciado en Psicología.

Máster en Psicología Jurídica y Mediación

Psicólogo del Punto de Encuentro Familiar de Castellón. Profesor colaborador Departamento de Ciencias Jurídicas y Sociales. Universidad Internacional de Valencia (VIU)

Santiago García Campá

Doctor en Derecho

Profesor Contratado Doctor. Departamento del Derecho del Trabajo y la Seguridad Social

Universitat Jaume I de Castellón

María Pilar Tormo Irún

Doctora en Psicología y Máster Internacional en Psicología Forense.

Profesora Ayudante Doctora. Directora del Máster Universitario en Prevención e Intervención Psicológica en Problemas de Conducta en la Escuela, Universidad Internacional de Valencia (VIU) Miembro del Grupo de Investigación "GI-VIU para el Estudio interdisciplinar de la Violencia" en las áreas: Violencia de género y Violencia y menores.

\section{resumen/abstract:}

En el presente artículo se analiza la normativa de los Puntos de Encuentro Familiar (en adelante, PEFs) en la Comunidad Valenciana y su actuación en casos de violencia de género teniendo también en cuenta la legislación de las Comunidades Autónomas de Islas Baleares, País Vasco, Cataluña y Aragón. Se identifican algunos problemas que surgen en los PEFs cuando la derivación procede de un procedimiento relacionado con la violencia de género, centrándonos concretamente en los dependientes de la Conselleria de Igualdad y Políticas Inclusivas de la Comunidad Valenciana. Las conclusiones extraídas ponen de manifiesto la extrema complejidad que entrañan estas situaciones, por lo que se recomienda 1) realizar una valoración sobre la necesidad real de la derivación al PEF por parte de los juzgados, 2) contar con formación específica en violencia de género por parte de los/as profesionales de los PEFs y 3) mejorar la coordinación con todos los agentes u organismos que intervienen en estos casos. También es necesario resolver los errores y demoras que se producen durante todo el recorrido judicial, desde que se toma la decisión de derivar un expediente al PEF hasta que empiezan a darse las visitas en el mismo.

In the present article we analyze the rules of the Family Meeting Points (hereinafter PEFs) in Valencian Community and their actions in cases of gender violence, having into account laws of the Autonomous Communities of Balearic Islands, Basque country, Catalonia and Aragon, too. Some problems are also identified in the PEFs when the referral 


\section{dossier}

La intervención con Menores Expuestos a Violencia de Género en los Puntos de Encuentro Familiar. Disfuncionalidades...

comes from a procedure related to gender violence, focusing specifically on the dependents of the Department of Equality and Inclusive Policies of the Valencian Community. The conclusions highlight the extreme complexity of these situations, consequently it is recommended that 1) an assessment of the real need for referral to PEF by courts is carried out, 2) professionals in PEFs have specific training in gender violence and 3) coordination among all actors or agencies involved in these cases is improved. It is also necessary to resolve errors and delays that occur throughout the judicial route, since the decision to refer a case to PEF is taken until the moment that visits start in it.

\section{palabras clave/keywords:}

Punto de Encuentro Familiar (PEF), Violencia de género, Menores, Comunidad Valenciana, Legislación Family Meeting Point (PEF in Spanish), Gender Violence, Children, Valencian Community, Legislation

\section{Introducción}

La problemática de los conflictos familiares cuenta cada vez con más recursos de intervención disponibles, especialmente en la Comunidad Valenciana. Entre ellos pueden ser destacados los Puntos de Encuentro Familiar o los Servicios de Mediación Familiar, en particular cuando intervienen en supuestos de violencia de género.

Los Puntos de Encuentro Familiar (en adelante, PEFs) son espacios neutrales que pretenden favorecer el derecho fundamental de niñas y niños a mantener las relaciones con sus familias cuando, en una situación de ruptura familiar o de interrupción de la convivencia familiar, son de difícil cumplimiento o se desenvuelven en un ambiente de alta conflictividad. En la mayor parte de las ocasiones, las personas usuarias del PEF tienen limitado su derecho a relacionarse con sus hijas o hijos tras una resolución judicial que impone la obligación de concurrir a dicho servicio, bien para realizar la recogida y posterior entrega de la persona menor, o bien para materializar in situ los encuentros en la propia sede del servicio, siendo tutelados dichos encuentros por el personal del PEF.

Desde que en 1994 se puso en funcionamiento el primer PEF en Valladolid, este servicio ha ido adquiriendo mayor protagonismo en nuestro contexto sociocultural. La demanda y necesidad de este servicio ha ido en aumento, habiéndose convertido en una alternativa válida a la vía judicial para resolver el conflicto familiar (Sacristán, 1999), especialmente en aquellas situaciones en que el progenitor no custodio presenta factores de riesgo que requieran supervisión (Sepúlveda y Serrano, 2000) y siempre debe preservar la integridad física y afectivo-emocional del menor (Morte y Lila, 2007).

En la Carta Europea de los Puntos de Encuentro para el mantenimiento de las relaciones entre los hijos y sus padres (Carta Europea, 2004), un documento marco con validez para todos los PEFs, se reconoce el vínculo de filiación, así como el interés y el derecho del menor en ver aseguradas la instauración y la continuidad de todas las relaciones necesarias para la construcción de su identidad.

A su vez, el Manual de uso práctico para los Puntos de Encuentro Familiar (Piñero, Vargas y Torres, 2009) describe detalladamente cómo funcionan los Puntos de Encuentro Familiar

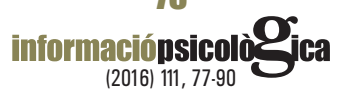




\section{dossier}

y ofrece orientaciones específicas sobre cómo deben relacionarse con las instituciones competentes para ofrecer un servicio integral y de calidad a las familias.

Más específicamente, el Documento Marco de mínimos para asegurar la calidad de los Puntos de Encuentro Familiar (Ministerio de Educación, Política Social y Deportes, 2008) establece los principios básicos necesarios para la intervención especializada en casos de violencia de género. Y estos principios reguladores del funcionamiento del PEF en los casos de violencia de género son ampliados en las Conclusiones de los IV Encuentros de magistrados y jueces de familia y asociaciones de abogados de familia y las VI Jornadas nacionales de magistrados, jueces de familia, fiscales y secretarios judiciales, celebradas en Valencia del 26 al 28 de octubre de 2009.

En estas situaciones pocos recursos sociales son más rentables social y económicamente que los PEFs, pues no deben minusvalorarse las repercusiones de todo tipo que genera una separación conflictiva, tanto en los progenitores como sobre todo en los hijos que lo sufren (Utrera, 2008).

La aprobación de la Ley Orgánica 1/2004, de 28 de diciembre, de medidas de protección integral contra la violencia de género, ha producido un incremento significativo del número de derivaciones de expedientes al PEF procedentes de los juzgados específicos de violencia contra la mujer; en algunos centros de la Comunidad Valenciana su número alcanza el 50 por ciento del total de derivaciones judiciales. De modo que los PEFs se han convertido en un recurso específico para el cumplimiento del régimen de visitas en los casos de violencia de género, singularmente cuando existen órdenes de protección para las madres.

En la actualidad, la mayor parte de las Comunidades Autónomas cuenta con un marco normativo que regula el régimen jurídico de los PEFs y sistematiza los objetivos que deben cubrir, también ante situaciones de violencia de género. Pero siguen siendo muchos los desafíos que todavía quedan pendientes por resolver, tal como señalan algunos trabajos previos (Morte y Lila, 2007). Como concluye el informe del Defensor del Menor de Andalucía (2012), los PEFs no solo deben contar con garantías jurídicas suficientes para desarrollar su labor de acuerdo con los derechos y obligaciones tanto de las personas que concurren al servicio -familiares y menores- como del personal que presta allí sus servicios, sino que también deben tener en cuenta las especiales circunstancias que concurren en las relaciones interpersonales cuando ha existido violencia de género en el seno familiar.

En el presente artículo se analiza, en primer lugar, la normativa de los PEFs en la Comunidad Valenciana y su actuación en casos de violencia de género, relacionando sus principales contenidos normativos con los adoptados en la legislación de las Comunidades Autónomas de Islas Baleares, País Vasco, Cataluña y Aragón; y, en segundo lugar, se identifican algunos problemas que surgen en los PEFs cuando la derivación procede de un procedimiento relacionado con la violencia de género, centrándonos concretamente en los dependientes de la Conselleria de Igualdad y Políticas Inclusivas de la Comunidad Valenciana (En la Comunidad Valenciana funcionan 18 PEFs, todos de titularidad de la Conselleria citada salvo el operativo en la ciudad de Valencia, de titularidad municipal). 


\section{Normativa autonómica sobre los Puntos de Encuentro Familiar}

La revisión normativa sobre los PEFs comprende su regulación legal en la Comunidad Valenciana (Ley 13/2008, de 8 de octubre; en adelante, LPEFCV), con una atención particular al tratamiento de su intervención en supuestos de violencia de género. El análisis legal integra una perspectiva comparada que también estudia la regulación efectuada en las Comunidades Autónomas de Islas Baleares (Decreto 57/2011, de 20 de mayo; en adelante, DPEFIB), País Vasco (Decreto 124/2008, de 1 de julio; en adelante, DPEFPV), Cataluña (Decreto 357/2011, de 21 de junio; en adelante, DPEFCat) y Aragón (Decreto 35/2013, de 6 de marzo; en adelante, DPEFAra). La legislación autonómica ha sido seleccionada teniendo en cuenta criterios de proximidad con la Comunidad Valenciana, salvo en el caso del País Vasco donde ha primado la calidad técnica de su regulación.

En la Tabla 1 se muestra el número de PEFs existentes en las Comunidades Autónomas estudiadas.

Tabla 1.- Puntos de encuentro en las Comunidades Autónomas estudiadas

\begin{tabular}{ll}
\hline $\begin{array}{l}\text { Comunidad } \\
\text { Autónoma }\end{array}$ & Número de PEFs \\
\hline Cataluña & 23 \\
C. Valenciana & 18 \\
Aragón & 6 \\
País Vasco & 5 \\
Illes Balears & 4 \\
\hline
\end{tabular}

Nota: Web de servicios sociales de los gobiernos autonómicos correspondientes.

Una primera diferencia entre los regímenes jurídicos autonómicos seleccionados se encuentra en el objeto regulado en cada Comunidad Autónoma: mientras que en Illes Balears y País Vasco la regulación se centra en la organización y funcionamiento de los PEFs por derivación judicial, la Comunidad Valenciana, Cataluña y Aragón abordan su objeto sin limitarlo a los supuestos de derivación judicial, aunque también la incluyen. La segunda diferencia radica en el instrumento legal empleado: un texto legislativo en el caso de nuestra Comunidad Autónoma y una norma reglamentaria en los demás.

La justificación de los Puntos de Encuentro Familiar es común, en la medida que reside en el art. 94 del Código Civil, que regula los efectos de la nulidad, separación y divorcio como materia de competencia estatal (art. 148.1.8. ${ }^{a}$ Constitución Española). Según este precepto, el progenitor que no tenga consigo a los hijos menores o incapacitados «gozará del derecho de visitarlos, comunicar con ellos y tenerlos en su compañía».

De acuerdo con el mismo precepto, el Juez determinará el tiempo, modo y lugar del ejercicio de este derecho, que podrá limitar o suspender si se dieren graves circunstancias que así lo aconsejen o se incumplieren grave o reiteradamente los deberes impuestos por la resolución judicial. 


\section{dossier}

En el mismo sentido, el art. 66 de la Ley Orgánica 1/2004, citada, modificado por la DF.3. ${ }^{a}$ de la Ley Orgánica 8/2015, de 22 de julio, de modificación del sistema de protección a la infancia y a la adolescencia, establece: «El Juez podrá ordenar la suspensión del régimen de visitas, estancia, relación o comunicación del inculpado por violencia de género respecto de los menores que dependan de él. Si no acordara la suspensión, el Juez deberá pronunciarse en todo caso sobre la forma en que se ejercerá el régimen de estancia, relación o comunicación del inculpado por violencia de género respecto de los menores que dependan del mismo. Asimismo, adoptará las medidas necesarias para garantizar la seguridad, integridad y recuperación de los menores y de la mujer, y realizará un seguimiento periódico de su evolución».

Según el Informe 2015 del Observatorio contra la violencia doméstica y de género del Consejo General del Poder Judicial, de las medidas judiciales de protección contenidas en los arts. 554 bis y ter Lecrim (reguladoras de la orden de protección) y otras medidas cautelares adoptadas en procesos por violencia de género, sólo el 3,5\% acordaron la suspensión del régimen de visitas, el 0,4\% la suspensión del régimen de potestad y el 5,9\% la suspensión de la guarda y custodia.

Si este precepto atiende fundamentalmente a los derechos de los progenitores, la Ley Orgánica 1/1996, de 15 de enero, de protección jurídica del menor (ajustada a la nueva situación de los menores en estos últimos años por la ley 26/2015), se centra en este para primar su interés sobre cualquier otro igualmente legítimo que pudiera concurrir (art. 2), lo que se traslada a los principios rectores de la actuación de los poderes públicos, quienes deben perseguir la supremacía del interés del menor y su integración social y familiar (art. 11.2). Se trata de un principio que también debe regir la actuación de los PEFs (art. 2 LPEFCV, art. 3.a) DPEFIB, art. 6.1 DPEFPV, art. 5.a) DPEFCat y art. 4.a) DPEFAra).

Entre los fines del PEF se encuentra facilitar el cumplimiento del régimen de visitas como un derecho fundamental del menor y el encuentro de los progenitores y demás familiares con el menor (art. 4 LPEFCV, art. 4 DPEFIB, art. 4 DPEFPV, art. 3 DPEFCat y art. 3 DPEFAra). En el caso de resolución judicial, el PEF tutela las visitas que tengan lugar en las dependencias donde presta servicio (art. 18.2 LPEFCV, art. 7.1.b) DPEFIB, art. 30 DPEFPV, art. 10.a) DPEFCat y art. 10.2.a) DPEFAra). En cambio, el PEF no intervendrá cuando el titular del derecho de visita se encuentre suspendido judicialmente del ejercicio del mismo (art. 7 LPEFCV).

El PEF tiene como objeto facilitar de forma profesional que los menores puedan mantener relaciones con sus familiares en supuestos de interrupción de la convivencia familiar, tal y como acontece en los supuestos de violencia de género en que la orden de protección contenga las medidas judiciales de carácter civil contenidas en el art. 544 ter.7 de la Ley de Enjuiciamiento Criminal (art. 2 LPEFCV, art. 2.a) DPEFIB, art. 2.1 DPEFPV y art. 2 DPEFAra). En tales supuestos, el órgano judicial es el que realiza la derivación al PEF (art. 9.1 LPEFCV, art. 9 DPEFIB, art. 18 DPEFPV, art. 8.1.a) DPEFCat y art. 14 DPEFAra). Paralelamente, en la orden de protección pueden acordarse medidas cautelares de carácter 


\section{dossier}

penal como el alejamiento o la suspensión de las comunicaciones con la víctima (art. 544 ter.6 Lecrim, art. 64 Ley Orgánica 1/2004), lo que, como se verá, obligará al PEF a adoptar medidas de seguridad especiales. De hecho, los PEFs deben coordinar el ejercicio de sus funciones y el desarrollo de su actividad especialmente con los juzgados derivantes y los servicios sociales de atención y protección a la mujer (art. 22.1 LPEFCV, art. 33 DPEFIB, art. 31 DPEFPV, arts. 5.b) y 30 DPEFCat y art. 26 DPEFAra).

Las medidas de carácter civil tienen una vigencia temporal de 30 días (art. 544 ter.7 Lecrim), cuya superación obligará al Juez competente a ratificarlas, modificarlas o dejarlas sin efecto. Existe controversia entre los profesionales sobre la oportunidad de la derivación al PEF durante los primeros 30 días. Por un lado, se estima muy difícil la intervención profesional en un espacio temporal tan breve, lo que se incrementa con la incertidumbre sobre la continuidad de las medidas. Por otro, teniendo en cuenta las especiales características de este tipo de casos, en los que ha existido violencia real, se considera una intervención en un escenario de crisis (Calzada, Sacristán y de la Torre, 2011), lo que incrementa de modo sobresaliente la dificultad de la intervención profesional.

El equipo profesional del PEF deberá contar como mínimo con un/a letrado/a, que será su coordinador/a; un/a psicólogo/a y un/a auxiliar administrativo/a. Puede completarse con un/a trabajador/a social y un/a educador/a social, de modo simultáneo o alternativo (art. 6 LPEFCV). En cambio, en las restantes Comunidades Autónomas estudiadas la regulación del equipo profesional varía de modo notable.

En la Comunidad Autónoma Balear se requiere la figura de la coordinación -quien debe contar alternativamente con la titulación de Derecho, Psicología, Pedagogía, Psicopedagogía, Trabajo Social o Educación Social; y, además, al menos doce meses de experiencia profesional en PEF- y el equipo técnico, formado por "diferentes perfiles profesionales" pero sin determinar su composición exacta (arts. 34 ss. DPEFIB). En el País Vasco el equipo profesional está estructurado por una coordinación, elegida entre quienes componen el equipo técnico, y dicho equipo, compuesto por un/a psicólogo/a, un/a trabajador/a social y un/a educador/a social como mínimo; (arts. 38 ss. DPEFPV). En Cataluña se establece un/a profesional con funciones de coordinación y un equipo de profesionales formado por psicólogos/as, educadores/as sociales y trabajadores/as sociales, cuyo número vendrá determinado por el volumen de actuaciones (arts. 22 ss. DPEFCat). En Aragón se contempla una regulación mínima que requiere la coordinación, quien puede compatibilizar sus funciones con las técnicas, y un equipo técnico multidisciplinar formado por titulados/as en psicología, trabajo social, educación social y derecho, sin concretar su número (art. 24 DPEFAra).

En el PEF se desarrollan varios tipos de intervención (art. 18 LPEFCV): supervisión de entrega y recogida de menores en ejercicio del derecho de visita fuera de sus instalaciones; tutela de las visitas que tengan lugar en sus instalaciones cuando así lo establece la resolución judicial; y puesta a disposición de las personas usuarias de los recursos humanos y materiales cuando las visitas no requieren supervisión directa o presencia continuada del equipo técnico. 


\section{dossier}

Las clases de intervención son tratadas de modo parecido en las restantes Comunidades Autónomas, si bien se aprecian las siguientes diferencias: en Islas Baleares se dividen en términos muy parecidos en entregas y recogidas, visitas tuteladas y no tuteladas y acompañamientos (art. 7 DPEFIB); en el País Vasco se refiere a intercambios en el centro, visitas tuteladas en el PEF y fuera de él, visitas no tuteladas y acompañamientos (art. 7 DPEFIB); en Cataluña se denominan servicios de intercambio, visitas tuteladas y visitas supervisadas (arts. 10 y 11 DPEFCat); y, finalmente, Aragón regula la entrega y recogida, las visitas supervisadas y las no supervisadas (art. 10 DPEFAra).

Con carácter general, la actuación del PEF comienza con la recepción de una ficha de derivación que debe acompañar el testimonio o copia íntegra de la resolución judicial donde se fija el régimen de las visitas y se acuerda la derivación al punto de encuentro familiar (art. 21 LPEFCV), aunque con ligeras diferencias entre Comunidades Autónomas (arts. 19 ss. DPEFIB, arts. 19 ss. DPEFPV, arts. 12 ss. DPEFCat, y arts. 14 ss. DPEFAra). Esta ficha debe estar acompañada del resto de documentación que sea pertinente, como informes psicosociales elaborados como pericial previa, otros informes psicológicos o sociales que figuren en el procedimiento judicial o, en su caso, la orden de alejamiento. La derivación será asignada a un profesional, quien abrirá un expediente, entrevistará por separado a las personas usuarias, efectuará una evaluación con la información recibida y elaborará un plan de intervención, tras el que comenzarán las visitas (arts. 21 ss. DPEFIB, arts. 21 ss. DPEFPV, arts.13 ss. DPEFCat y arts. 13 ss. DPEFAra). Debe tenerse en cuenta que en la Ley valenciana las actuaciones posteriores a la recepción de la ficha de derivación no se regulan expresamente, de acuerdo con los arts. 21 ss. LPEFCV. La actuación finalizará por resolución judicial, excepto en los supuestos de suspensión por resolución igualmente judicial o a instancia motivada del equipo profesional (arts. 27 y 28 LPEFCV, arts. 29 y 30 DPEFIB, art. 33 DPEFPV, arts. 18 y 20 DPEFCat y arts. 18 y 19 DPEFAra).

Durante la prestación del servicio debe garantizarse la seguridad de cualquier persona usuaria del PEF, lo que requiere el establecimiento de un protocolo de vigilancia con las Fuerzas y Cuerpos de Seguridad del Estado (art. 30 LPEFCV). En los supuestos en que exista una orden de protección deben adoptarse medidas de seguridad especiales para garantizar su vigilancia y protección. Las medidas deben formalizarse mediante un protocolo de actuación en coordinación con las Fuerzas y Cuerpos de Seguridad del Estado. Además, en algunas normas autonómicas se difiere temporalmente el momento de la entrega del menor y de su recogida para evitar la coincidencia entre agresor y víctima, incluso el acompañamiento del menor por un profesional desde su domicilio hasta el PEF (art. 32 DPEFIB y art. 30 DPEFPV). Si los profesionales del PEF advierten la existencia de un riesgo para la integridad de las personas, darán aviso a la autoridad correspondiente (art. 29 LPEFCV, art. 42 DPEFIB, arts. 30 y 37 DPEFPV, art. 30 DPEFCat y art. 20 DPEFAra). En algunos casos se reconoce la posibilidad de solicitar a las autoridades judiciales o policiales que las visitas tuteladas cuenten con acompañamiento policial (art. 42 DPEFIB, art. 37 DPEFPV) y de suspender la intervención para asegurar la integridad física o psíquica de la víctima y del menor en sus dependencias (art. 29 DPEFIB, art. 26 DPEFPV, art. 18 DPEFCat, 20 DPEFAra). 


\section{Problemáticas asociadas al uso de un Punto de Encuentro Familiar de la Comunidad Valenciana: El caso de los menores expuestos a violencia de género}

A continuación se describe el funcionamiento de los PEFs dependientes de la Conselleria de Igualdad y Políticas Inclusivas de la Generalitat Valenciana, así como diversas incidencias que se suscitan en la práctica cotidiana de un Punto de Encuentro Familiar, y que pueden afectar a los derechos de los menores expuestos a violencia de género que han de acudir allí para mantener contacto con su progenitor.

La titularidad del servicio corresponde a la Administración Valenciana, aunque la misma desde la propia creación del servicio ha ido concediendo la contratación del servicio a distintas entidades.

Cada PEF está conformado por un/a psicólogo/a y un/a auxiliar administrativo/a, que prestan sus servicios 20 horas semanales, y un/a Letrado-Coordinador/a, que tiene a cargo 4 PEFs, con un horario de 40 horas semanales. Desde mediados de julio de 2015, existe la figura de 4 psicólogos/as itinerantes de refuerzo, los cuales prestan sus servicios en los PEFs en los que se les requiere, siendo su horario de 20 horas semanales. Existe un LetradoCoordinador Autonómico encargado de supervisar el servicio, con un horario de 40 horas semanales.

El horario de los PEFs es de 20 horas semanales: Lunes, miércoles y viernes de 17 a 20h; Sábados de 10 a 14h y de 17 a 20h; Domingos de 17 a $21 \mathrm{~h}$.

Es conveniente para un mejor entendimiento del entorno a tratar, describir el mismo en etapas, las cuales comprenderán desde que una familia es derivada al PEF hasta que se abandona el mismo.

Las etapas se desglosarán de forma cronológica, siendo las siguientes: (1) Derivación, (2) Entrevistas de recepción, (3) Estudio del expediente y programación del inicio, (4) Inicio. Primeras visitas, (5) Desarrollo de las visitas y (6) Finalización de la Intervención del PEF.

\section{Derivación:}

La derivación de una familia en la que ha existido violencia de género se inicia tras la realización del juicio rápido. Si hay menores, en este estadio se debe establecer, a la par de las medidas penales, unas medidas civiles que regulen la relación de estos con su progenitor.

Cuando el juzgado deriva un expediente al PEF, junto con la documentación judicial, ha de ir aparejada una ficha de derivación en la cual figurarán, entre otros, los datos de las partes y el tipo de visita a realizar. Hay ocasiones en que ya desde el juzgado existe una demora en el envío de dicha documentación, dándose algunos casos en los que son los propios progenitores quienes acuden al PEF antes de que este tenga conocimiento del caso.

En otras ocasiones la documentación llega incompleta y hay que solicitarla a la entidad derivante, por lo que se sigue produciendo un retraso que incluso puede llegar a ser de meses. Una vez el expediente ha llegado completo al PEF, dependerá de cada centro su fecha de inicio, ya que algunos PEF pueden tener lista de espera debido al volumen que manejan -lo cual evidentemente dilatará nuevamente el inicio de las visitas.

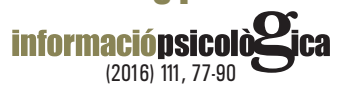




\section{Entrevistas de recepción:}

Siguiendo con el protocolo del PEF, se realizan entrevistas de recepción a las partes. Lógicamente ambos progenitores son citados en diferentes horarios y/o días de modo que no coincidan en las instalaciones.

Estas entrevistas sirven para advertir cuál es la dimensión del conflicto y, sobre todo, el estado del menor y la predisposición del mismo en cuanto a relacionarse con su progenitor. También se valora si es conveniente que el menor acuda antes del inicio de las visitas, si se evalúa que este tiene miedo a iniciar las visitas en el PEF o que la información que se le ha proporcionado no se corresponde con la realidad. Cuando se cita a los menores para que acudan antes de iniciar visitas, se observa que estos quedan más tranquilos, de modo que el día de inicio de las visitas acuden con menos ansiedad.

Es frecuente, ya en la entrevista, que el progenitor trate de averiguar información de la otra parte a través del PEF, ya que la orden de alejamiento y la ruptura de las relaciones hacen que el único medio de información pueda ser el centro. De hecho, en muchas ocasiones durante la entrevista ambas partes preguntan por el otro. En definitiva, se busca obtener información, siendo distintos los objetivos de cada progenitor. Por tanto, el personal técnico ha de estar preparado tanto directamente -ante posibles preguntas formuladas por el progenitor- como de modo indirecto, para no dejar al alcance ni a la vista del progenitor cualquier tipo de documento que tenga que ver con la otra parte, o bien para llevarse consigo la documentación en caso de abandonar el despacho ante alguna urgencia o imprevisto.

\section{Estudio del expediente y programación del inicio:}

Con la información extraída de las entrevistas de recepción más la aportada por el órgano derivante se analiza el expediente. En ocasiones, el PEF es contactado por profesionales de otros servicios como casas de acogida, servicios especializados de atención a la familia e infancia (SEAFI), Centro mujer 24h, técnicos municipales adscritos a las concejalías de la mujer, que atienden a usuarias con violencia de género y/o a los menores donde se hallan usuarias del PEF, aportando información y/o solicitándola.

Tras el análisis del expediente se fija el inicio de las visitas, lo cual se traslada a las partes y al órgano derivante.

\section{Inicio. Primeras visitas:}

La primera visita suele ser la más crítica debido, por un lado, a la ansiedad anticipatoria con la que acude el menor al centro y, por otro lado, al concepto que pueda tener de su progenitor tras lo sucedido, tanto por si ha presenciado episodios de violencia como por lo que se le haya dicho durante el tiempo que no ha tenido contacto con su progenitor.

Muestran los estudios que los/as niños/as que afrontan los conflictos entre sus progenitores tomando parte del mismo, es decir, implicándose ellos también, presentan más problemas de conducta. Sin embargo, cuando recurren a estrategias de afrontamiento que les hacen tomar distancia del conflicto presentan una mejor adaptación (Cantón, Cortés y Justicia, 2000; De la Torre, 2005).

A ello también confluye el miedo que en muchas ocasiones manifiesta la víctima de violencia de género a encontrarse con su agresor en los aledaños del PEF -miedo que suele

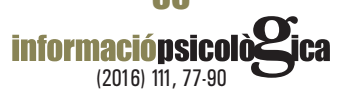


ser inoculado al menor. Aunque la llegada al PEF de ambos progenitores se programa en distintos momentos para evitar coincidencias y estos son informados previamente en las entrevistas de recepción, no quita que muchas veces se manifieste dicho temor.

Una vez la progenitora y el menor se hallan en una sala y el progenitor en otra, se evalúa el estado del menor por si hubiese que intervenir con el mismo. Tras ello se acompaña al menor a la sala donde le espera su progenitor.

Si el tipo de visita a realizar en el PEF es Tutelada con Supervisión (lo cual conlleva que el personal técnico esté presente en toda la visita), este debe estar muy pendiente de que la visita no sirva para obtener información del menor, siendo frecuente que el progenitor indague en cuestiones tales como si siguen viviendo en el mismo domicilio, si su madre trabaja, si tiene novio, si tiene nuevo número de teléfono, o incluso tratar de transmitir algún tipo de información a la ex-pareja usando al menor como correo.

También suele suceder -aunque en las entrevistas se les recomienda que no es oportunoque en la primera o primeras visitas acudan acompañando al PEF familiares de ambas partes, quedándose estos en los aledaños del mismo. Pero la motivación es distinta en cada caso. Los familiares de la progenitora buscan darle más seguridad al acudir a un lugar donde se halla su agresor. En cambio, los familiares del progenitor acuden al presentárseles una oportunidad, o tal vez la única, de poder ver al menor. En otras ocasiones simplemente tratan de prolongar el conflicto al estar disconformes con lo resuelto por el juzgado.

Estas situaciones generan mucha tensión, por lo que es preciso hacer ver a las partes que estas conductas no son buenas para el menor, ya que se ve sometido a una presión y a la disyuntiva de "elegir" si saluda o no a la familia del progenitor.

No debemos perder la perspectiva de que los menores se hallan en un proceso adaptativo y lo que más influye en la adaptación de los menores a la separación o divorcio de sus progenitores es la respuesta de estos al estrés, más que el estrés en sí mismo (Cantón et al., 2000; De la Torre, 2005).

Obviamente el grado de afección del menor ante la ruptura de sus progenitores, amén de la causa que lo originó, dependerá de varios factores. Uno de los más importantes será la edad del mismo, teniendo en cuenta que la horquilla en que un menor puede acudir al PEF es desde los 3 meses a los 18 años (art. 80 Ley 15/2005).

En términos generales ante una separación, las reacciones más habituales son las siguientes, en función de su edad (Calzada et al., 2011):

\section{Desde el nacimiento hasta la edad preescolar:}

Los menores muestran confusión ante la dificultad de entender lo que sucede y miedo a ser abandonados. Suelen entender la separación como algo temporal, se pueden dar conductas agresivas y regresivas.

\section{Primeros años escolares (6-8 años):}

La marcha del progenitor ausente puede producir gran tristeza al ser interpretada como una pérdida y mostrar deseos de que regrese. Suele haber episodios de enfado del menor hacia ambos progenitores o sobre el que considera culpable de la separación.

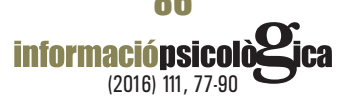




\section{dossier}

Años escolares posteriores (9-12 años):

Los menores comienzan a construir su realidad, tratando de hallar un culpable. Es en estos años cuando toman parte de modo más activo por uno de los progenitores. Se suelen dar conductas de enfado, dolencias psicosomáticas, agresividad y conductas de exigencia buscando que se haga lo que él desea.

Adolescencia (más de 12 años):

Los cambios naturales que se dan a estas edades (fisiológicos, desarrollo del cuerpo, búsqueda de la propia identidad) llevan a que, en los casos de separaciones, los menores se sientan más capaces de tomar sus propias decisiones acerca de las visitas con el progenitor titular del derecho de visitas. En algunas ocasiones, es habitual que el menor quiera evitar toda relación con el mismo, bien como muestra de su disconformidad por la situación existente o porque se ha generado una alianza con el progenitor titular de la guarda y custodia. Con frecuencia tanto los menores como los progenitores manifiestan que cuando el menor cumpla 12 años dejará de acudir al PEF a ver al progenitor con quien tiene visitas.

También cabe tener en cuenta que hacer comparecer a los menores en sede judicial conlleva unos perjuicios (desasosiego, sentimientos de culpa, conflicto de lealtades) que se deberían evaluar antes de tomar una decisión al respecto, ya que las consecuencias repercutirán en las visitas en el PEF (Utrera, 2008).

A este respecto, el artículo 9 de Ley Orgánica 1/1996, de 15 de enero, de protección jurídica del menor señala que el menor tiene derecho a ser oído, tanto en el ámbito familiar como en cualquier procedimiento administrativo o judicial en que esté directamente implicado y que conduzca a una decisión que afecte a su esfera personal, familiar o social. Respecto a la audiencia de los menores en los procesos de familia, que es donde cobra total protagonismo dicho Derecho, el artículo 770.4 de la Ley de Enjuiciamiento Civil señala que, si el procedimiento fuere contencioso y se estime necesario de oficio o a petición del fiscal, partes o miembros del equipo técnico judicial o del propio menor, se oirá a los hijos menores o incapacitados si tuviesen suficiente juicio y, en todo caso, a los mayores de doce años.

Retomando lo que puramente respecta al régimen de visitas, en muchos casos de violencia de género se constata la necesidad de crear nuevas formas de relacionarse entre el padre y el menor, ya que se aprecian juegos de tipo violento entre ambos, así como en el trato hacia los juguetes del centro y/o el resto de usuarios y personal del PEF. Se observan patrones de comportamiento rígidos y escasas manifestaciones afectivas, todo ello unido a sentimientos de enfado y rabia generados por la experiencia vivida, y que es necesario atajar.

Gran parte de la tarea en el PEF consiste en reconducir las posiciones de los progenitores desde el conflicto hacia procesos constructivos en los que el menor se beneficie de una relación cordial de los progenitores. Pero aún así, los primeros contactos de los menores con el PEF muestran en estos el anhelo de que sus progenitores sigan juntos. Este deseo puede permanecer de meses a años e incluso si no se aborda dejará secuelas en los mismos. Y es que muchos menores, en función de su nivel evolutivo, prefieren ver a sus padres juntos aunque la relación no funcione y pese al riesgo que entraña para los mismos cuando hay violencia de género. 
Otra de las observaciones más frecuentes es encontrarnos con menores que forman parte activa del conflicto, al cual han sido llevados por los progenitores de modo consciente o inconsciente. En estos menores se advierten episodios ansiosos y/o depresivos, como reacción al dilema de elegir entre uno u otro progenitor.

Por lo tanto, resulta necesario apoyar a los menores en este tránsito ya que, como previamente se ha señalado, debido a su estado evolutivo no ven la separación como la mejor solución tras el fracaso de sus progenitores si no como el fin de lo que hasta ahora habían conocido, con la incertidumbre de lo que vendrá.

El itinerario vital de cada familia también condiciona la adaptación de los menores, teniendo en el PEF cada vez más casos de menores que han vivido varias rupturas previas de uno u otro progenitor, así como de otros hijos que ya han sido testigos de algunas situaciones por las que puede que pasen ellos.

\section{Desarrollo de las visitas:}

Si todo lo señalado anteriormente se resuelve en las visitas y paralelamente con las partes, se logrará, no solo restablecer la relación paterno-filial y el vínculo con el menor, sino también mejorar su competencia en el ejercicio de la crianza y la coparentalidad.

Obviamente no es fácil, puesto que en el caso de los progenitores también se observan sentimientos encontrados. Es difícil de asimilar, por un lado, para la progenitora, que el menor estime a la persona que le ha hecho daño y, por parte del progenitor, que el menor estime a su madre después de la denuncia interpuesta en su contra por violencia de género.

En muchas ocasiones las intervenciones tienen lugar en momentos de crisis, cuando todavía están en estado de shock o comienzan a reorganizarse y acuden al PEF obligados por un tercero, lo cual para unos es tomado como una injerencia y para otros como una fortuna, ya que permite restablecer las relaciones interrumpidas, aún en un entorno ajeno como es el PEF. Esto ocurre especialmente en los casos de violencia de género, donde la llegada al PEF es inmediata, por lo que el conflicto está aún muy latente, añadido a la incertidumbre del recorrido que tendrá el procedimiento penal.

En todo este tránsito los menores no quedan al margen, y será de vital importancia resolver los conflictos que se vayan presentando tanto con los progenitores como en la relación de estos con los menores.

\section{Finalización de la Intervención del PEF:}

Como ya se ha apuntado, en los casos de violencia de género los expedientes están más supeditados si cabe al recorrido judicial que tengan. Es decir, además de las medidas civiles, que serán las que marcarán las relaciones paterno-filiales, paralelamente existe un procedimiento penal que puede dilatarse en el tiempo, observándose ciertos casos en los que dichas demoras han alcanzado hasta 4 años. Estas demoras pueden deberse a varios factores, como la carga de trabajo que soporta cada juzgado y/o los recursos interpuestos a instancias superiores, y que puede dar lugar a la absolución del caso o a su condena e ingreso en un centro penitenciario. 


\section{dossier}

Por otro lado, existen expedientes en los que solo han de acudir al PEF el tiempo que dure la orden de protección, finalizando la intervención del PEF cuando termina esta.

\section{Conclusiones}

Una de las situaciones más complejas que debe afrontar la intervención en los PEFs tiene que ver con las rupturas derivadas por violencia de género, siendo consideradas por algunos especialistas como intervenciones en crisis (Calzada et al., 2011).

La revisión de la normativa sobre el PEF en la Comunidad Valenciana ha mostrado un esquema de intervención prácticamente coincidente con el de otras Comunidades revisadas, con alguna diferencia en el objeto de regulación, la descripción de sus fases o la composición del equipo de intervención. En todos los casos existen previsiones generales en materia de seguridad y medidas específicas en el caso de la existencia de una orden de protección contra la violencia de género. Estas medidas deben formalizarse mediante un protocolo de actuación en coordinación con las Fuerzas y Cuerpos de Seguridad del Estado. En otras Comunidades también se prevén otras actuaciones como la entrega y recogida diferidas de las/os menores, su acompañamiento hasta el domicilio del otro progenitor e incluso la suspensión de la visita o de la intervención para asegurar la integridad física o psíquica de la víctima y del menor en sus dependencias.

La praxis profesional muestra la conveniencia de que los juzgados hagan un primer juicio de valoración sobre la necesidad real de la derivación al PEF, con la finalidad de no colapsar el recurso.

Los/as profesionales que trabajan en el PEF han de poseer la formación adecuada para las intervenciones que realizarán, siendo imprescindible la formación específica en violencia de género si se tiene en cuenta el volumen creciente de derivaciones acordadas por resolución judicial, especialmente en la Comunidad Valenciana.

Si la coordinación con todos los agentes u organismos debe caracterizar la intervención del equipo profesional en los PEFs, su intensidad debe incrementarse con los órganos judiciales o los centros específicos de atención a las víctimas de violencia de género. Los errores deben ser resueltos y las demoras advertidas en el presente trabajo desde que se toma la decisión judicial de derivar un expediente al PEF hasta que las visitas comienzan.

\section{Referencias}

Asociación Española de Abogados de Familia (2009). Conclusiones de los IV Encuentros de magistrados y jueces de familia y asociaciones de abogados de familia y las VI Jornadas nacionales de magistrados, jueces de familia, fiscales y secretarios judiciales, celebradas en Valencia del 26 al 28 de octubre de 2009. Disponible en http:// ayudaafamiliasseparadas.es/archivo/archivo/IV\%20ENCUENTRO\%20MAGISTRADOS.pdf

Calzada, E., Sacristán, M. I. y de la Torre, J. (2011). La intervención psicosocial en los puntos de encuentro familiar. Valladolid, España: Ministerio de Sanidad y Política Social.

Cantón, J., Cortés, M. del R. y Justicia, M. D. (2000). Conflictos matrimoniales, divorcio y desarrollo de los hijos. Madrid: Pirámide. 


\section{dossier}

Carta Europea de los Puntos de Encuentro para el mantenimiento de las relaciones hijos-padres (2004). Ginebra. Disponible en http://www.fedepe.org/pdf/Carta Europea PEF.pdf

Decreto de los servicios técnicos de punto de encuentro (Decreto 357/2011, de 21 de junio). Diario Oficial de la Generalitat de Catalunya, № 5906, 2011, 23 junio.

Decreto de modificación del Decreto regulador de los puntos de encuentro familiar por derivación judicial en la Comunidad Autónoma del País Vasco (Decreto 239/2011, de 22 de noviembre). Boletín Oficial del País Vasco, № 228, 2011, 1 diciembre.

Decreto por el que se aprueba el Reglamento de funcionamiento de los Puntos de Encuentro Familiar en Aragón (Decreto 35/2013, de 6 de marzo, del Gobierno de Aragón). Boletín Oficial de Aragón, № 53, 2013, 15 marzo.

Decreto por el cual se establecen los principios generales de organización y funcionamiento de los puntos de encuentro familiar por derivación judicial (Decreto 57/2011, de 20 de mayo). Boletín Oficial de las Islas Baleares, № 081, 2011, 2 junio.

Decreto regulador de los puntos de encuentro familiar por derivación judicial en la Comunidad Autónoma del País Vasco (Decreto 124/2008, de 1 de julio). Boletín Oficial del País Vasco, № 149, 2008, 7 agosto.

Defensor del Menor de Andalucía (2012). Menores expuestos a violencia de género: Víctimas con identidad propia. Sevilla: Defensor del Menor de Andalucía. Disponible en http://www.defensordelmenordeandalucia.es/node/2245

De la Torre, J. (2005). Las relaciones entre padres e hijos después de las separaciones conflictivas. Apuntes de psicología, 2311), 101-112.

Ley Orgánica de medidas de protección integral contra la violencia de género (Ley Orgánica 1/2004, de 28 de diciembre). Boletín Oficial del Estado, № 313, 2004, 29 diciembre.

Ley Orgánica de modificación del sistema de protección a la infancia y a la adolescencia (Ley Orgánica 8/2015, de 22 de julio). Boletín Oficial del Estado, № 175, 23 julio.

Ley Orgánica de modificación del sistema de protección a la infancia y a la adolescencia (Ley Orgánica 26/2015, de 28 de julio). Boletín Oficial del Estado, № 180, 2015, 29 julio.

Ley Orgánica de protección jurídica del menor, de modificación del Código Civil y de la Ley de Enjuiciamiento Civil (Ley Orgánica 1/1996, de 15 de enero). Boletín Oficial del Estado, № 15, 1996, 17 enero.

Ley reguladora de los Puntos de Encuentro Familiar de la Comunidad Valenciana (Ley 13/2008, de 8 de octubre, de la Generalitat). Boletín Oficial de la Generalitat Valenciana, №5871, 2008, 16 octubre.

Ministerio de Educación, Política Social y Deportes (2008). Documento Marco de mínimos para asegurar la calidad de los Puntos de Encuentro Familiar. Madrid: Ministerio de Educación, Política Social y Deportes. Disponible en http:// www.msssi.gob.es/ssi/familiasInfancia/docs/2009-marco-minimos-asegurar-calidad-pef.pdf

Morte, E. y Lila, M. (2007). La alternativa al conflicto: Punto de Encuentro Familiar. Intervención Psicosocial, 16(3), 289-302.

Piñero, J., Vargas, F. y Torres, E. (2009). Puntos de Encuentro Familiar: manual de uso práctico. CEE Limencop. Disponible en http://www.fundacionsaludinfantil.org/ libro.htm

Sacristán, M. L. (1999). El lugar de encuentro. Un recurso en la gestión de los conflictos familiares. Ponencia I Congreso de Mediación Familiar. Valencia.

Sepúlveda, A. y Serrano, F. (2000). Punto de Encuentro familiar: Un recurso social alternativo. Apuntes de Psicología, $18(2 y 3), 375-382$.

Utrera G., J. L., (2008). Jornada problemes emergents entorn dels menors i les famílies en l'ambit civil. Barcelona, 14 de maig. Disponible en http://docplayer.es/5520943-Jornada-problemes-emergents-entorn-dels-menors-i-lesfamilies-en-l-ambit-civil.html 